\title{
SELECCIÓN DE PORTAFOLIOS DE INVERSIÓN INCLUYENDO EL EFECTO DE ASIMETRÍA: EVIDENCIA CON ACTIVOS DE LA BOLSA MEXICANA DE VALORES
}

Miguel Flores-Ortega* Lilia Alejandra Flores Castillo** Angelica Paredes Gómez

(Recibido: abril 2014/Aceptado: septiembre 2014)

\section{Resumen}

En este trabajo se presenta la evidencia empírica de incorporar el efecto de la asimetría del rendimineto de los activos en el modelo de portafolio para la selección de los activos y su participación en la integración un portafolio de inversión. Se realiza la revisión teórica de la metodología utilizada y se presenta el contraste de los resultados del modelo de Markowitz que utiliza únicamente la media y la varianza media-varianza y el modelo que incorpora la asimetría.

La metodología que se utiliza plantea un problema de optimización multiobjetivo, que selecciona el portafolio de inversión que minimiza la función multiobjetivo, con lo cual se logra la optimización simultánea de los objetivos particulares de media, varianza y asimetría. Los resultados muestran que es posible disminuir la probabilidad de rendimientos negativos y pérdidas en el caso de seleccionar el portafolio con mayor asimetría positiva. La modelo valida todas las posibilidades de selección del nivel de aversión al riesgo, rendimiento y asimetría, el enfoque que se presenta tiene la ventaja de ser flexible y la selección de los activos se expresa de forma

\footnotetext{
* Profesor-investigador, de la Escuela Superior de Economía del Instituto Politécnico Nacional; $<$ mfo@prodigy.net.mx>.

** Alumnos de doctorado, de la Escuela Superior de Economía del Instituto Politécnico Nacional.
} 
matemática en un espacio definido por la varianza, la expectativa de rendimiento y su asimetría. El trabajo presenta evidencia empírica con activos de la Bolsa Mexicana de Valores.

Palabras clave: portafolio de inversión, optimización, teoría de la decisión estadística.

Clasificación JEL: C44, C61, G11.

\section{Abstract}

This paper shows the empirical evidence of the effect of asymmetry of return assets in the portfolio selection and integrates their participation in an investment portfolio. The theoretical review of the methodology is performed and contrasts a results Markowitz model that uses only the mean and variance and mean-variance model incorporating asymmetry effect. The methodology propose a problem of multi-objective optimization, which selects the investment portfolio that minimizes the multi-objective function, whereby the simultaneous optimization of the specific aims of mean, variance and skewness is achieved. The results show that it is possible to decrease the probability of negative returns and losses in the case of selecting the portfolio more positively skewed. The model validates all selection options level of risk aversion, performance or asymmetry, the approach presented has the advantage of being flexible and selection of assets is expressed mathematically in a space defined by the variance, expected return and asymmetry. The paper presents empirical evidence of the Mexican stock exchange assets.

Keywords: portfolio investment, optimization, statistical decision theory. JEL classification: C44, C61, G11:

\section{Introducción}

En el mercado financiero se reúnen agentes económicos denominados inversionistas los cuales realizan operaciones de compra o venta de activos financieros con el objetivo de obtener una ganancia o beneficio económico. El tema de inversión económica siempre ha llamado la atención de los investigadores por su complejidad, la teoría de portafolio establece las bases científicas que permiten seleccionar el conjunto de activos y el monto que 
se debe invertir en cada uno de ellos para maximizar la función de utilidad del inversionista.

El trabajo seminal sobre portafolios de inversión corresponde a Harry Markowitz (1952), quien encontró que la correlación entre el rendimiento de entre parejas de activos seleccionados es imporante para determinar el riesgo de invertir en el portafolio; el resultado de incorporar el efecto de la correlación al evaluar el riesgo de un portafolio permite reducir el riesgo conforme aumenta el númeo de activos que se seleccionan.

El modelo original de Markowitz utiliza la media y la varianza como parámetros de decisión para conformar un portafoloio de inversión y determinar la proporción que se debe invertir en cada uno de los activos seleccionados; la función de utilidad del portafolio adquiere una representación cuadrática bajo el supuesto de que el comportamiento estadístico del rendimiento de los activos corresponde a la distribución normal. Una critica a los supuestos de Markowitz la presentó Fama (1965) y Arditti (1971) quienes afirman que el comportamiento estadístico de los rendimientos en la mayoría de las series financieras no cumplen con los supuestos de normalidad.

En los trabajos de Rubinstein (1973), Hanoch y Levy (1970) se argumenta que si la distribución se aleja de la distribución normal, la función de utilidad cuadrática presenta limitaciones porque sólo considera la utilidad marginal positiva para un rango acotado y es necesario incorporar otros parámetros de la distribución para tomar en cuenta los efectos asimétricos en el cálculo del riesgo sobre todo cuando se presentan colas anchas en la distribución.

EL objetivo de la investigación es buscar la evidencia empírica del efecto de incorporar el parámetro de asimetría en la selección del portafolio y como afecta la utilidad del inversionista. Al incorporar el parámetro de asimetría cómo variable de decisión en la selección de un portafolio de inversión se logra mayor flexibilidad en la selección de los activos y una mejor aproximación a los deseos del inversionista

La idea central de investigación es analizar el problema de optimización multiobjetivo que considera diferentes combinaciones de preferencias del inversionista sobre la media, la varianza y la asimetría, una aportación de este enfoque es mostrar que el modelo es muy flexible y se puede utilizar de diferentes maneras dependiendo de las preferencias del inversionista. En el análisis de selección de portafolio es importante seleccionar aquel que muestre asimetría positiva, dado que al incrementar la asimetría aumenta la probabilidad de rendimientos positivos y decrece la probabilidad de rendimientos negativos. 
La investigación presenta evidencia empírica del efecto de incorporar la asimetría en la selección de un portafolio de inversión, se utilizaron diez acciones de empresas que cotizan en la Bolsa Mexicana de Valores y se seleccionaron por ser las acciones que tienen mayor participación en el índice de precios y cotizaciones de la bolsa, el horizonte de estudio corresponden a datos diarios del periodo del 2 de enero de 2009 al 15 de noviembre de 2013.

Al plantear el problema de selección de un portafolio con múltiples objetivos se debe solucionar de forma simultánea para todos los objetivos, se parte del trabajo de Lai (1991) que utiliza un modelo de programación matemática para optimizar el portafolio, la función objetivo corresponde a un polinomio con las variables de los objetivos individuales, la solución debe satisfar de forma simultánea todos los objetivos y se incorporan las preferencias del inversionista por medio de un ponderador para cada uno de los objetivos.

El trabajo se organiza en seis secciones; la primera es la introducción al tema, el objetivo de investigación y la estruccuta del trabajo; en la segunda sección se presenta la revisión de la literatura y un análisis conceptual; se dedica la tercera seccion para abordar la metodología de optimización del portafolio multiobjetivo, para incorporar el efecto de la asimetría en la selección de activos para un portafolio de inversión; en la cuarta sección se presenta el análisis de los datos de los activos seleccionados de la bolsa mexicana de valores; en la sección cinco se muestra la integración de portafolios multiobjetivo y se realiza el análisis de resultados; por último, en la sexta sección se incorporan las conclusiones del trabajo.

\section{Revisión de la literatura y un análisis conceptual}

Los antecedentes de la teoría de portafolio se encuentran en el trabajo de Hicks (1935), quien explica que en toda inversión, existen factores de riesgo que se deben considerar y son el reflejo de la incertidumbre sobre las pérdidas o las ganancias que se pueden presentar, por lo que la teoría de portafolio de inversión utiliza el rendimiento esperado y su varianza como criterio para la selección del portafolio de inversión y la proporción de los activos seleccionados.

De forma paralela, Tobin (1958) trabajó en el problema del portafolio y coincidió con la hipótesis de Markowitz (1952), pero en lugar de utilizar en el análisis la varianza, considera la desviación estándar y establece que el riesgo asociado al rendimiento de un portafolio se mide por la desviación estándar 
de los rendimientos, que es una medida de la dispersión de los rendimientos posibles con respecto al valor esperado y la raíz cuadrada de la varianza.

Tobin (1958) determinó que cuando se observa una desviación estándar alta significa que existe la probabilidad de grandes desviaciones, tanto positivas como negativas, una baja desviación estándar significa baja probabilidad de grandes desviaciones. En el caso extremo, una desviación estándar igual a cero indica la seguridad de recibir el rendimiento esperado. Por lo tanto, un portafolio de inversión de varianza alta ofrece al inversionista la posibilidad de obtener grandes ganancias de capital a un precio de oportunidades equivalentes a las pérdidas de capital y un portafolio de bajo valor de varianza protege al inversionista de pérdida de capital, y también indica pocas posibilidades de ganancias inusuales.

Markowitz (1952) demostró de forma geométrica, que existe un conjunto de portafolios eficientes e ineficientes. En la figura 1, se define una curva de isomedia como el conjunto de todos los portafolios para un rendimiento esperado. Del mismo modo se define una línea de isovarianza donde converge el conjunto de todos los portafolios con una varianza de rendimientos y el nivel de exposición de riesgo. En el centro del sistema se encuentra el portafolio que minimiza la varianza, se marca este punto con $X$. El punto de la línea de isomedia que la varianza toma como valor mínimo corresponde al punto en el que la línea de isomedia es tangente a una curva de isovarianza. A medida que una curva de isovarianza se aleja del punto $X$, la varianza el riesgo se incrementa.

Figura 1

Análisis geométrico de selección de un portafolio eficiente

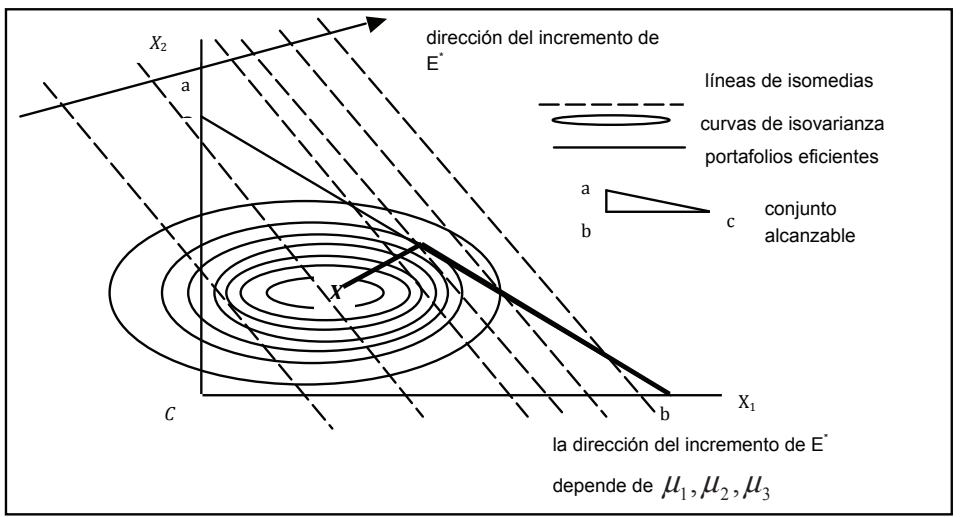

Fuente: Portfolio Selection, Markowitz (1952, p. 85). 
El análisis geométrico de Markowitz (1952) utiliza líneas de isomedias, curvas de isovarianzas y el supuesto de que cuando una curva de isovarianza se aleja más del punto $X$ la varianza se incrementa, lo que tiene lógica con respecto a la función de utilidad del inversionista.

En el trabajo de Marschak (1938) que considera el análisis de la teoría monetaria, la teoría general del valor y la ecuación de cambio como instrumento para explicar los problemas monetarios, determinó que para el análisis de los problemas de inversión es necesario ampliar el concepto de los gustos humanos y no sólo tomar en cuenta la aversión al riesgo del inversionista, sino también el deseo por adquirir riqueza entre otros rasgos del comportamiento. Marschak estableció que existe una función de utilidad que permite expresar los gustos del inversionista con la ecuación de la distribución del rendimiento de la combinación de activos que cumple con la condición que maximiza la función de utilidad.

El problema principal del análisis de portafolio de inversión es determinar el conjunto de portafolios factibles que conforman la frontera eficiente, $y$ para cada nivel de riesgo existe un conjunto de portafolios que satisfacen las preferencias, de forma tal que la frontera eficiente establece la región factible del inversionista el límite inferior corresponde al mínimo rendimiento y el portafolio de máximo rendimiento. Sharpe (1963) determinó una forma de simplificar los cálculos, su contribución principal a la teoría de portafolio fue plantear el modelo diagonal por medio del cual se puede resolver el problema de análisis de inversión mediante la solución del problema de maximización que representa la ecuación 1.

$$
\begin{gathered}
\text { mazimizar: } \lambda E-V \\
\text { donde: } E=\sum_{i=1}^{N+1} X_{i} A_{i} \\
V=\sum_{i=1}^{N+1} X_{i}^{2} Q_{i} \\
\text { sujeto } a: X_{i} \geq 0 \text { para todo } \text { ide } 1 \text { a } N \\
\sum_{i=1}^{N} X_{i}=1 \\
\sum_{i=1}^{N} X_{i} B_{i}=X_{n+1}
\end{gathered}
$$

El modelo de la ecuación 1 es el más sencillo, se denomina diagonal porque la matriz de varianza-covarianza, está llena de valores y se expresa 
como una matriz con elementos diferentes a cero sólo a lo largo de la diagonal mediante la inclusión de una matriz ampliada $(n+1)$. Con este procedimiento se reduce el número de cálculos necesarios para resolver el problema de selección de los activos del portafolio.

\subsection{Supuestos económicos para la formulación del modelo de portafolio}

El primer supuesto para la formulación del modelo asume que la distribución de los rendimientos de los activos es normal con parámmetros $\mu=0$ y $\sigma^{2}$ constante en el tiempo, se asume que los rendimientos son independientes entre sí y la varianza es aleatoria.

La construcción inicial del portafolio acepto que todo inversionista es un agente económico adverso al riesgo que busca maximizar la función de utilidad al final de cada periodo, se admite que existe un conjunto grande de posibilidades de inversión y que el último activo corresponde al activo libre de riesgo. Para el modelo se considera que todos los activos son negociables y perfectamente divisibles con responsabilidad limitada a su valor; condición que permite una representación continua, se consigue que la tasa de endeudamiento sea única e igual a la tasa de rendimiento que corresponde al activo libre de riesgo, se asume que el mercado de capitales es perfecto, lo que significa que no existen impuestos a las ganancias ni costos de transacción.

\subsection{Rendimiento y riesgo del portafolio de inversión}

La teoría del portafolio considera que las decisiones de inversión dependen de la relación riesgo-rendimiento de los activos que se seleccionan, se asume que el rendimiento de un activo es el cambio de valor que se registra en un periodo con respecto a su valor inicial.

$$
R_{i}=\frac{\Delta \text { valor }}{\text { valor }_{\text {inicial }}}=\frac{\text { valor }_{\text {final }}-\text { valor }_{\text {inicial }}}{\text { valor }_{\text {inicial }}}
$$

El rendimiento del portafolio se expresa por :

$$
\mathrm{R}_{\mathrm{p}}=W^{T} R=\sum_{i=1}^{n} w_{i} R_{i}
$$

Donde $w_{i}$ representa el porcentaje de riqueza que se invierte en el activo $i$ y $R_{i}$ es el rendimiento esperado del activo $i$. El modelo no se considera ventas 
en corto, por lo que todos los ponderadores deben ser no negativos $w_{i} \geq 0 \mathrm{y}$ la suma de las ponderaciones debe ser igual a la unidad,

$$
\sum_{i=1}^{n} w_{i}=1
$$

El riesgo de la inversión en el portafolio se expresa por medio de la varianza del rendimiento del portafolio, que representa la dispersión de los rendimientos alrededor de la media. Se asume que un portafolio es eficiente si la varianza asociada al rendimiento esperado $\mu$ es mínima con respecto al conjunto de portafolios factibles que tienen el mismo rendimiento esperado, también un portafolio con varianza $\sigma^{2}$ es eficiente si el rendimiento esperado $\mu$ es máximo para el conjunto de portafolios factibles que tienen la misma varianza, que corresponde a la expresión 4, para el activo $i$.

$$
\sigma_{i}^{2}=\sum_{i=1}^{n}\left[R_{i}-E(R)\right]^{2}
$$

para el portafolio

$$
\sigma_{\mathrm{p}}^{2}=W^{T} \sum W=\sum_{i=1}^{n} w_{1}^{2} \sigma_{i}^{2}+\sum_{i=1}^{n} \sum_{j=1}^{n} w_{i} w_{j} \sigma_{i j} \quad \text { para } i \neq j,
$$

donde:

$\sigma_{i}^{2}$ representa la varianza del activo $i$,

$\sigma_{i j}$ corresponde a la covarianza entre el activo $i$ y el $j$,

$\Sigma$ representa la matriz de varianza covarianza para $\mathrm{n}$ activos,

$W^{T}$ representa al vector transpuesto de los pesos de los activos en el portafolio.

Un portafolio con máxima varianza no necesariamente lleva al máximo rendimiento, por lo que el inversionista que selecciona un portafolio eficiente para obtener mayor rendimiento debe asumir un mayor nivel de riesgo, la estrategia de inversión busca el portafolio que maximiza la expectativa de rendimiento y minimiza la varianza.

De acuerdo con el planteamiento de la solución del problema de optimización multiobjetivo el portafolio óptimo que se selecciona es el que minimiza la función multiobjetivo, con lo cual se logra la optimización simultánea de los valores de la media, la varianza y la asimetría, reduce la probabilidad de que se presenten rendimientos negativos y se incurra en pérdidas y se obtiene el portafolio con mayor asimetría positiva. 
La aportación de Samuelson (1970) muestra que cuando la serie no tiene un comportamiento de distribución normal y se incorpora el efecto del tercer momento estadístico, la selección del portafolio presenta un sesgo con respecto al valor del momento más significativo; por lo tanto, se debe asumir que la incorporación de todos los momentos de la distribución son importantes para la toma de decisiones de inversión y no se deben despreciar. El estudio de Levy y Sarnat (1972) denota que el efecto de la asimetría de la serie es importante para explicar el comportamiento de los activos y el rendimiento del portafolio.

Simkowitz y Beedles (1978) presentaron una extensión al trabajo de Markowitz (1952) con la inclusión del tercer momento que ha sido debatida, encontraron que no es deseable la diversificación cuando se quiere seleccionar un portafolio que tome en cuenta los tres primeros momentos estadísticos porque se reduce el efecto de la asimetría.

El trabajo de Kane (1982) presenta evidencia de que la selección del tercer momento influye en la determinación del portafolio óptimo y la selección de activos riesgosos, el impacto de incluir una función que utilice el efecto de la asimetría en las preferencias del inversionista es deseable. Si los componentes son negativos o pequeños la pérdida de la asimetría por la diversificación es más rápida que la reducción de la varianza.

Desde el punto de vista de Lai (1991), al incluir la asimetría en la selección de un portafolio de inversión se presenta un conflicto de objetivos dado que el inversionista desea maximizar tanto el rendimiento como la asimetría y minimizar el riesgo, para resolver el dilema propone formular un problema multiobjetivo con ayuda de un modelo de programación lineal que utiliza un polinomio para definir la función multiobjetivo. Konno y Suzuki (1995) extendieron el análisis de forma geométrica y lograron trazar una superficie eficiente en el espacio de media-varianza-asimetría.

De forma similar, el trabajo de Lai (1991) es el de Chunhachinda, Dandapani, Hamid y Prakash (1997), que presentan un modelo con una función multiobjetivo polinomial para la selección de un portafolio con asimetría que considera 14 índices de mercados accionarios en el entorno internacional. Los resultados del estudio empírico muestran que la incorporación de la asimetría para la toma de decisión de inversión del portafolio causa un cambio importante en la construcción del portafolio óptimo, sobre todo porque los inversionistas estarán en una mejor situación de alcanzar mejores expectativas de rendimiento. 
Leung, Daouk y Chen (2001) también investigaron la utilidad y eficacia del método multiobjetivo, la evaluación del desempeño se mide al utilizar técnicas de pronósticos que incluyen cambios en las preferencias del inversionista sobre el rendimiento. La varianza y la asimetría, en su estudio, concluyen que la metodología permite derivar más beneficios que otros métodos, cuando el ambiente de inversión se vuelve volátil e inestable.

Con la finalidad de observar las oportunidades de diversificación Prakash, Chang y Pactwa (2003) extendieron el análisis de la selección de portafolio al incluir rendimientos mensuales de 17 índices accionarios internacionales de mercados emergentes en Latinoamerica y mercados desarrollados de Estados Unidos y Europa, la evidencia del estudio demuestra que la incorporación de la asimetría en las preferencias del inversionista origina un portafolio diferente al considerar otras restricciones y valores de la asimetría de los activos; el resultado de los portafolios eficientes para el modelo media-varianza-asimetría son mejores que los que se obtienen con el modelo de media-varianza, un hallazgo adicional corresponde a identificar que en los portafolios de países desarrollados, la diversificación reduce la asimetría con mayor velocidad que en países emergentes y también se identificó que los portafolios eficientes en promedio presentan asimetría negativa.

En el estudio de Zuluaga y Cox (2010) se propone una metodología para incorporar el efecto de la asimetría en los portafolios que determinan la frontera eficiente con el objetivo mejorar la relación riesgo-rendimiento. La metodología busca tomar en cuenta la posibilidad de que se presenten grandes pérdidas en relación al portafolio óptimo y controlar el efecto del lado izquierdo de la distribución conforme se aleja de la normal. La incorporación de una restricción de probabilidad de pérdida dentro del problema de optimización logra acotar y acercar el extremo de la cola izquierda al valor medio.

El trabajo de Usta y Kantar (2011) presenta un enfoque multiobjetivo que analiza un modelo que toma en cuenta: la media, la varianza, la asimetría y la entropía, para establecer la selección de los activos que conforman el portafolio de inversión; uno de los resultados relevantes del estudio revela que la inclusión de la asimetría de la muestra mejora significativamente la selección del portafolio conforme la distribución de los rendimientos se aleja de la distribución normal. 


\section{Metodología de optimización multiobjetivo; incorporar el efecto de la asimetría en la selección de un portafolio de inversión}

El modelo de selección de portafolio multiobjetivo que propuso Lai (1991), para incorporar el efecto de la asimetría en la selección de un portafolio de inversión, requiere de las consideraciones esenciales en la que se sustenta la teoría de portafolios; se asume que todo inversionista es un agente económico adverso al riesgo que busca maximizar la función de utilidad al final de cada periodo, además considera que existe un conjunto grande de posibilidades de inversión y el último activo corresponde al activo libre de riesgo, todos los activos son negociables y perfectamente divisibles con responsabilidad limitada a su valor; condición que permite una representación continua, se asume que la tasa de endeudamiento es única e igual a la tasa de rendimiento que corresponde al activo libre de riesgo, el mercado de capitales es perfecto, que significa que no existen impuestos a las ganancias ni costos de transacción.

La solución original del problema de selección de portafolio la propuso Makowitz (1952) al plantear la metodología media-varianza, consiste en determinar las proporciones de riqueza $w$ que maximizar el rendimiento y minimizar el riesgo del portafolio lo que se logra con una adecuada diversificación de los activos. La solución original del problema del portafolio se otiene al solucionar la expresión 1.6 que representa el modelo que minimiza el riesgo y el rendimiento se incorpora como una restricción:

Sujeto a:

$$
\begin{gathered}
\min \sigma_{p}^{2}=W^{T} V W \\
E\left(R_{p}\right)=W^{T} R \\
W^{T} I=1 \quad W \geq 0
\end{gathered}
$$

La selección del portafolio que considera la media-varianza como variables de decisión se aplica cuando la distribución de los rendimientos de los activos sigue una distribución normal. El argumento de Fama (1965) que indica que la distribución de los rendimientos de activos financieros no tiene un comportamiento normal, sugiere incluir el análisis de la asimetría dentro de la estructura de las preferencias del inversionista en la selección del portafolio de inversión y utilizar la solución de un problema de optimización multiobjetivo, mediante el cual se o-determina la proporción que se debe invertir en cada activo del portafolio, para que se maximice el rendimiento y la asimetría, al mismo tiempo que se minimice el riesgo de la inversión. 
El planteamiento de Lai (1991) parte de la determinación de los modelos individuales para calcular el rendimiento, la asimetría y la varianza. Los modelos del portafolio con objetivos individuales los describen las ecuaciones 7,8 y 9, para cada caso.

$$
\begin{aligned}
& \text { Rendimiento del portafollio }=W^{T} R=\sum_{i=1}^{n} w_{i} R_{i} \\
& \begin{aligned}
\text { Varianza del portafolio } & =W^{T} \Sigma W \\
= & \sum_{i=1}^{n} w_{1}^{2} \sigma_{i}^{2}+\sum_{i=1}^{n} \sum_{j=1}^{n} w_{i} w_{j} \sigma_{i j} \text { para } i \neq j
\end{aligned} \\
& \begin{aligned}
\text { Asimetría del portafolio } & =E\left[W^{T}(\tilde{R}-\bar{R})\right]^{3} \\
= & \sum_{i=1}^{n} w_{i}^{3} S_{i}^{3}+3 \sum_{i=1}^{n}\left(\sum_{j=1}^{n} w_{i}^{2} w_{j} S_{i i j}+\sum_{j=1}^{n} w_{i} w_{j}^{2} S_{i j}\right) \text { para } i \neq j
\end{aligned}
\end{aligned}
$$

En las ecuaciones $\sigma_{i}^{2}$ representa la varianza del activo $i ; \sigma_{i j}$ es la covarianza entre las variables aleatorias $i$ y $j$ que describen el movimiento conjunto entre las variables; $\Sigma$ define la matriz de varianza covarianza para los rendimientos de $n$ activos; $s_{i}^{3}$ es asimetría del rendimiento del activo $i, W^{T}$, indica al vector transpuesto de los pesos de las posiciones del portafolio.

La ecuación 5 permite optimizar los objetivos individuales de cada variable que maximizan el rendimiento, la asimetría y minimizan el riesgo; en cada caso las otras variables no participan en el proceso de ajuste ni de optimización; en cada modelo se determinan los pesos que logran el objetivo particular.

$$
\left.\begin{array}{ll}
\text { maximizar } & R(w)=W^{T} R \\
\text { minimizar } & V(w)=W^{T} \Sigma W \\
\text { maximizar } & S(w)=E\left[W^{T}(R-\bar{R})\right]^{3}
\end{array}\right\} \text { sujeto } a W^{T} I=1, W \geq 0
$$

Los resultados que se obtienen a partir de los modelos individuales, evidencian que existen conflictos de intereses, al considerar un objetivo en particular, por lo que es necesario buscar una alternativa que permita conciliar los objetivos de forma simultánea. 
La optimización multiobjetivo es la técnica que busca satisfacer de forma simultánea un conjunto de objetivos de manera que en conjunto se encuentre la mejor solución. La función multiobjetivo que se propone es un polinomio que suma cada una de las funciones de utilidad con lo que se busca el máximo global, esta función se puede enriquecer si se adicionan los parámetros de las preferencias de los inversionistas en relación a cada objetivo. La estructura del modelo corresponde a los valores de las tres funciones individuales: $R(w), V(w)$ y $S(w)$, se utilizan los multiplicadores $\lambda$ que establecen las preferencias del inversionista sobre la media, varianza y asimetría de los rendimientos y se incorporan a la ecuación 6 .

$$
\begin{gathered}
\text { minimizar } Z=-\lambda_{1}(W \bar{R})+\lambda_{2}\left(W^{T} \Sigma W\right)-\lambda 3\left(E\left[W^{T}(R-\bar{R})\right]^{3}\right) \\
W^{T} I=1, W \geq 0
\end{gathered}
$$

La solución del problema de optimización se logra al minimizar la función multiobjetivo, de tal forma que se determina la cantidad que se debe adquirir de cada activo, consecuentemente se obtiene el portafolio que maximiza la función de utilidad del inversionista al incluir las preferencias para cada uno de los parámetros e incorporar el nivel de aversión al riesgo del inversionista.

\section{Análisis de los datos de los activos seleccionados de la Bolsa Mexicana de Valores}

En esta sección se analiza la selección de datos que se utilizó para el análisis; la muestra corresponde a un conjunto de 10 acciones que cotizan en la Bolsa Mexicana de Valores, la selección de los activos se realizó en función a la participación en el índice de precios y Cotizaciones (IPyC) de la bolsa, se asume como indicador el precio de cierre de cada activo y el periodo abarca desde el 2 de enero de 2009 al 15 de noviembre de 2013 y se determina el rendimineto diario.

El cuadro 1 presenta el conjunto de activos seleccionados, muestra el nombre, la clave de pizarra y el porcentaje de participación en el índice. 


\section{Cuadro 1}

Participación de las acciones en el IPyC

\begin{tabular}{|l|l|c|}
\hline \multicolumn{1}{|c}{ Empresa } & \multicolumn{1}{c|}{ Emisora } & Peso relativo $\%$ \\
\hline America móvil & AMXL & $15.93 \%$ \\
\hline Fomento Económico Mexicano & FEMSA UBD & $10.68 \%$ \\
\hline Grupo Financiero Banorte & GFNORTE & $8.12 \%$ \\
\hline Wal-Mart & WALMEX V & $7.32 \%$ \\
\hline Grupo televisa & TLEVISA CPO & $6.7 \%$ \\
\hline Grupo México & GMEXICO B & $6.77 \%$ \\
\hline Cementos Mexicanos & CEMEX CPO & $6.8 \%$ \\
\hline Grupo industrial Alfa & ALFA A & $5.6 \%$ \\
\hline Coca Cola Femsa & KOF L & $3.5 \%$ \\
\hline Kimberly y Clark & KIMBER A & $2.37 \%$ \\
\hline
\end{tabular}

Fuente: elaboración propia con datos del IPyC.

Los datos históricos de los precios de los activos seleccionados se utilizan para calcular los rendimientos, la matriz de varianza-covarianza y los momentos estadísticos. La metodología parte del analisis de la normalidad de la distribución de los rendimientos de los activos que integran el portafolio de inversión.

\section{Gráfica 1}

Tendencia de los precios de las acciones

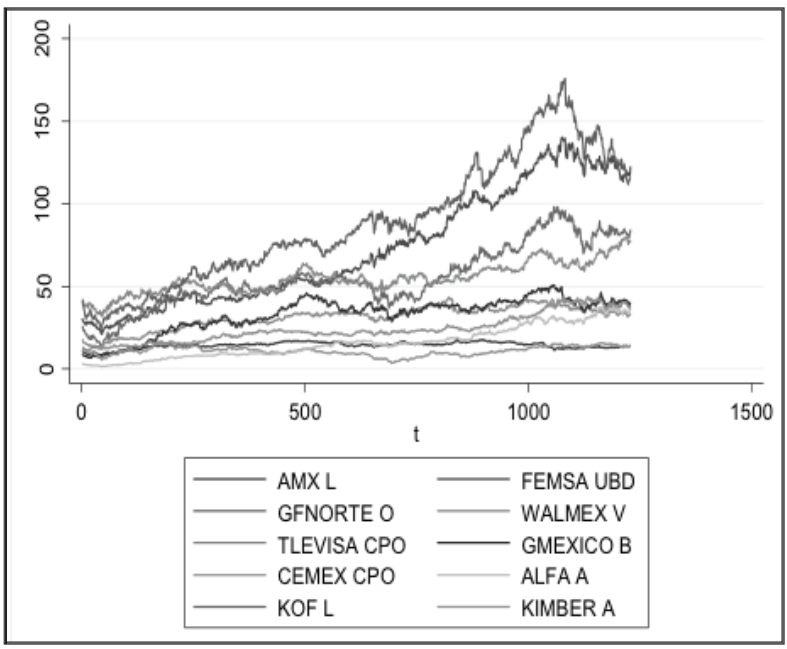

Fuente: elaboración propia con datos del IPyC. 
En la gráfica 1 se presenta la evolución de los precios de cada uno de los activos, se observa una fuerte tendencia positiva y un cambio sistemático en la media.

Los rendimientos de cada activo se calculan de forma logarítmica:

$$
r_{t}=\ln \left(\frac{P_{t}}{P_{t-1}}\right)
$$

La gráfica 2 presenta el comportamiento del rendimiento de las acciones seleccionadas, las series de rendimientos se mueven en torno a una media constante igual a cero, sin embargo la varianza de los rendimientos no se mantiene constante y cambian con el tiempo, se observan periodos en los que la volatilidad es menor y periodos en los que es mayor lo que representa un clásico agrupamiento de la volatilidad que se denomina heteroscedasticidad.

\section{Gráfica 2}

Rendimientos de las acciones

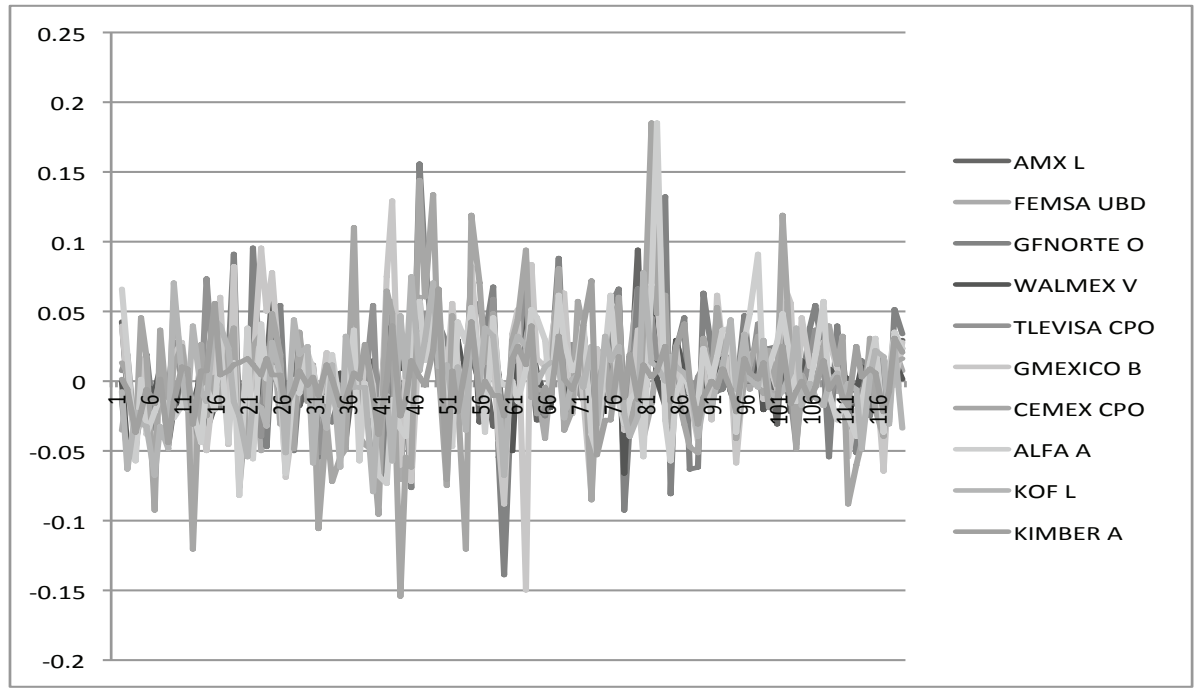

Fuente: elaboración propia con datos del IPyC.

En el cuadro 2 se presentan los valores de la media y la varianza de los rendimientos de cada uno de los activos. En la primera columna se observa que la acción ALFA A es el activo que tiene el rendimiento medio más alto, seguido por GFNORTE O y FEMSA UBD, mientras que AMXL presenta el rendimiento más bajo. 


\section{Cuadro 2}

Descripción estadística y prueba de normalidad para cada acción

\begin{tabular}{|l|c|c|c|c|c|}
\hline & Rendimiento & Varianza & Curtosis & $\begin{array}{c}\text { Coeficiente } \\
\text { de asimetría }\end{array}$ & Jarque Bera \\
\hline \hline AMX L & 0.000410 & 0.000248 & 5.04779 & -0.130668 & $1294(0.00)$ \\
\hline FEMSA UBD & 0.001266 & 0.000278 & 7.55926 & 0.037818 & $2896.58(0.00)$ \\
\hline GFNORTE O & 0.001266 & 0.000612 & 4.73531 & 0.46162 & $1179.150 .00)$ \\
\hline WALMEX V & 0.000655 & 0.000249 & 3.96552 & -0.43606 & $834.93(0.00)$ \\
\hline $\begin{array}{l}\text { T L E V I S A } \\
\text { CPO }\end{array}$ & 0.000660 & 0.000266 & 4.8226 & 0.637952 & $1261.04(0.00)$ \\
\hline GMEXICO B & 0.001551 & 0.000564 & 4.27078 & 0.222088 & $933.62(0.00)$ \\
\hline CEMEX CPO & 0.000617 & 0.000936 & 5.41983 & 0.260327 & $1501.940 .00)$ \\
\hline ALFA A & 0.002347 & 0.000501 & 5.61605 & 0.640957 & $1681.78(0.00)$ \\
\hline KOF L & 0.001025 & 0.000368 & 2.24237 & -0.137665 & $257.94(0.00)$ \\
\hline KIMBER A & 0.001029 & 0.000290 & 1.88318 & 0.288221 & $196.09(0.00)$ \\
\hline
\end{tabular}

Fuente: elaboración propia con datos del IPyC.

En la columna dos se incluye la varianza para cada unos de los activos, la varianza mínima corresponde a la acción AMXL y el activo con la varianza más alta y por lo tanto el más riesgoso fue CEMEX CPO. En el caso de los valores de la asimetría y la curtosis que representan el tercer y cuarto momento de la distribución de los rendimientos. Los activos de FEMSA UBD, GFNORTE O, TLEVISA CPO, GMEXICO B, CEMEX CPO, ALFA A y KIMBER A denotaron una asimetría positiva, la distribución está sesgada a la derecha lo que indica que la probabilidad de obtener un rendimiento por encima de la media es más probable que obtener un rendimiento por debajo de la media; mientras que AMXL, WALMEX V y KOF L manifestaron asimetría negativa. Por lo que se refiere a la curtosis, a excepción de KOF L y KIMBER A, los demás activos mostraron un exceso de curtosis.

Las características de la media, la varianza y la asimetría de los activos individuales indicaron que la distribución de los rendimientos no sigue una distribución normal. Las distribuciones de los rendimientos son asimétricas y la mayoría son leptocúrticas, los coeficientes de asimetría son negativos o positivos, su coeficiente de curtosis es mayor que el de una distribución normal, lo que implica colas más anchas y apuntalamiento en su distribución; esto significa que la probabilidad de obtener resultados muy lejos de la media es más común que lo pronosticado por una distribución normal. 
Con el objetivo de concluir, si la distribución de los rendimientos sigue una distribución normal, se realizó una prueba de normalidad, se empleó la prueba Jarque-Bera con un valor crítico de 5.99 para un nivel de significancia de $5 \%$, se establecieron las siguientes hipótesis:

$\mathrm{H}_{\mathrm{o}}=$ los rendimientos están distribuidos normalmente

$\mathrm{H}_{\mathrm{a}}=$ los rendimientos no se distribuyen normalmente

En el cuadro 2 se encuentra que la probabilidad asociada a los valores de la prueba Jarque-Bera es menor a 5\%; en el caso del valor del estadístico JB calculado todos los activos superan el valor crítico; se concluyó que en todos los casos se rechaza la hipótesis de que los rendimientos siguen una distribución normal.

\section{Integración de portafolios multiobjetivo y análisisi de resultados}

El argumento de aceptar que una serie no sigue una distribución normal evidencia la importancia de analizar la incorporación de la asimetría en la determinación de un portafolio de inversión; en particular si la presencia de asimetría se presenta en todas las distribuciones de los activos analizados.

Para el análisis e integración del portafolio multiobjetivo, la muestra de rendimientos de los activos se dividió en periodos de un año y para cada año se seleccionó un portafolio de inversión.

En el análisis primero se consideró la selección de un portafolio que sigue el criterio del modelo de Markowitz de media-varianza, selección se realizó con la incorporación de la asimetría y se realizó el análisis comparativo.

El estudio se complementó al incluir el análisis para diferentes niveles de preferencia y verificar la sensibilidad del modelo, la estructura de preferencias que se consideraron son las siguientes: $(1,0,0),(0,1,0)$, $(0,0,1),(1,1,0),(1,1,1),(2,1,0),(2,2,1),(1,2,1),(1,1,2),(1 / 2,0,1 / 2),(0,1 / 2,1 / 2)$, $(1 / 2,1 / 4,1 / 4),(1 / 4,1 / 2,1 / 4),(1 / 4,1 / 4,1 / 2),(1 / 3,1 / 3,1 / 3)$.

\section{Selección del portafolio con el modelo de Markowitz de media y varianza}

En el proceso de selección de portafolio, al considerar el modelo de Markowitz, se estructuró un conjunto de portafolios anuales, cada portafolio denotó diferentes valores para los parámetros de la media y la varianza, en el cuadro 3 se muestran los resultados para cada uno de los portafolios. 


\section{Cuadro 3}

Portafolios anuales considerando el modelo de media-varianza

\begin{tabular}{|l|l|l|l|l|c|}
\hline & \multicolumn{1}{|c|}{2009} & \multicolumn{1}{c|}{$\mathbf{2 0 1 0}$} & \multicolumn{1}{c|}{$\mathbf{2 0 1 1}$} & \multicolumn{1}{c|}{$\mathbf{2 0 1 2}$} & $\mathbf{2 0 1 3}$ \\
\hline \hline Rendimiento & 0.00188558 & 0.00102296 & 0.00104846 & 0.00137292 & 0.00041282 \\
\hline Riesgo & 0.00018092 & $6.3864 \mathrm{E}-05$ & 0.00014794 & $4.4434 \mathrm{E}-05$ & 0.00010384 \\
\hline Asimetría & 0.14556368 & -0.43388873 & 0.08213708 & -0.30889435 & 0.0042798 \\
\hline $\begin{array}{l}\text { Coeficiente de } \\
\text { variación }=(\sigma / \bar{x})\end{array}$ & 7.1334 & 7.8121 & 11.6008 & 4.8552 & 24.6843 \\
\hline \hline
\end{tabular}

Fuente: elaboración propia con datos del IPyC.

El portafolio que se selecciona mediante el modelo media-varianza, el que corresponde al año 2012, es el portafolio que minimizó el riesgo, con una varianza de $4.4434 \mathrm{E}-05$ y un coeficiente de variación de 4.8552 , este portafolio minimiza el riesgo y presenta un mayor grado de diversificación. A través del proceso de optimización se asignó una proporción de capital a todos los activos que conforman dicho portafolio a excepción de CEMEX $\mathrm{CPO}$ que mostró una participación nula, en el cuadro 4 se observa que el rango de diversificación se determinó entre 0.033 y 0.203 .

\section{Cuadro 4}

Composición de los pesos del portafolio óptimo seleccionado con el modelo de media-varianza

\begin{tabular}{|l|r|r|r|r|r|}
\hline \multicolumn{2}{|c}{2009} & 2010 & 2011 & 2012 & \multicolumn{1}{c|}{2013} \\
\hline$X_{\text {AMX L }}$ & 0 & 0.16244169 & 0.07825128 & 0.12787052 & 0.13287111 \\
\hline$X_{\text {FEMSA UBD }}$ & 0.12348956 & 0.14850374 & 0.41324834 & 0.1071043 & 0.14403102 \\
\hline$X_{\text {GFNORTE O }}$ & 0 & 0.05416205 & 0 & 0.0977222 & 0.02271416 \\
\hline$X_{\text {WALMEX V }}$ & 0.2555886 & 0.16658119 & 0.11480461 & 0.11547424 & 0 \\
\hline$X_{\text {TLEVISA CPO }}$ & 0.11598985 & 0.00300803 & 0 & 0.15495324 & 0.26734886 \\
\hline$X_{\text {GMEXICO B }}$ & 0.03773491 & 0 & 0 & 0.03270177 & 0 \\
\hline$X_{\text {CEMEX CPO }}$ & 0 & 0 & 0 & 0 & 0.15381592 \\
\hline$X_{\text {ALFA A }}$ & 0.03104953 & 0.21215633 & 0 & 0.08746483 & 0.05927768 \\
\hline$X_{\text {KOFL }}$ & 0.16770117 & 0.13091277 & 0.22227522 & 0.20309693 & 0.08010418 \\
\hline$X_{\text {KIMBER A }}$ & 0.26844639 & 0.1222342 & 0.17142055 & 0.07361197 & 0.13983707 \\
\hline
\end{tabular}

Fuente: elaboración propia con datos del IPyC. 


\section{Selección del portafolio con el modelo media-varianza-asimetría}

La selección del portafolio óptimo con el modelo media-varianza-asimetría se determina al obtener la solución del problema de optimización multiobjetivo, la estructura de la función multiobjetivo corresponde a un polinomio que incorpora los valores de las tres funciones individuales para la maximización del rendimiento $R(w)$, minimización del riesgo $V(w)$ y maximización de la asimetría $S(w)$, representadas en las ecuaciones 2, 3 y 4; para integrar el grado de preferencia entre los objetivos se utilizan los multiplicadores $\lambda$ que representan las preferencias del inversionista sobre la media, varianza y asimetría de los rendimientos, en este caso el problema de optimización multiobjetivo se resuelve con las preferencias $(1,1,1)$. El portafolio que se selecciona es el que obtiene el valor más pequeño al minimizar la función multiobjetivo:

$$
\text { minimizar } Z=\lambda_{1}(W \bar{R})+\lambda_{2}\left(W^{T} \Sigma W\right)-\lambda_{3}\left(E\left[W^{T}(R-\bar{R})\right]^{3}\right)
$$

los resultados se muestran en el cuadro 5.

\section{Cuadro 5}

Solución del problema multiobjetivo

para el caso de las preferencias $(1,1,1)$

\begin{tabular}{|c|c|c|c|c|c|}
\hline & 2009 & 2010 & 2011 & 2012 & 2013 \\
\hline Rendimiento & 0.00200679 & 0.00108922 & 0.00101206 & 0.00169243 & 0.0005278 \\
\hline Riesgo & 0.00020295 & 7.6238E-05 & 0.00016879 & 5.9673E-05 & 0.00012134 \\
\hline Asimetría & 0.31933886 & 0.46223742 & 0.28835367 & 0.15294366 & 0.29049812 \\
\hline $\begin{array}{l}\text { Valor de la fun- } \\
\text { ción de utilidad }\end{array}$ & 1.40450674 & 1.4617535 & 1.168719 & 1.55847642 & 1.55364142 \\
\hline
\end{tabular}

Fuente: elaboración propia con datos del IPyC.

Para un inversionista que incorpora un grado de preferencia por la media, la varianza y la asimetría, el portafolio seleccionado de acuerdo a los resultados obtenidos al minimizar la función multiobjetivo, el correspondiente al año 2011, es el portafolio que minimizó dicha función con un valor mínimo de 1.168719. En el cuadro 6 se muestra la composición de los pesos del portafolio. 


\section{Cuadro 6}

Composición de los pesos del portafolio para el caso de las preferencias $(1,1,1)$

\begin{tabular}{|c|c|c|c|c|c|}
\hline & 2009 & 2010 & 2011 & 2012 & 2013 \\
\hline$X_{\mathrm{AMXL}}$ & 0.02757011 & 0.06173401 & 0 & 0 & 0 \\
\hline$X_{\text {FEMSA UBD }}$ & 0.19675474 & 0 & 0.46362865 & 0.20779183 & 0.15219483 \\
\hline$X_{\text {GFNORTE O }}$ & 0 & 0.07455827 & 0 & 0.11705534 & 0.12069797 \\
\hline$X_{\text {WALMEXV }}$ & 0.13489365 & 0.14335734 & 0 & 0 & 0 \\
\hline$X_{\text {TLEVISA CPO }}$ & 0.1859193 & 0.20613691 & 0.06291419 & 0.16743562 & 0.35259752 \\
\hline$X_{\text {GMEXICO B }}$ & 0.01156182 & 0 & 0 & 0.05807366 & 0 \\
\hline$X_{\text {CEMEX CPO }}$ & 0 & 0 & 0 & 0 & 0.10929237 \\
\hline$X_{\text {ALFA A }}$ & 0.12058669 & 0.22878953 & 0 & 0.1352935 & 0.06548169 \\
\hline$X_{\text {KOF L }}$ & 0.11749714 & 0.13162674 & 0.051084 & 0.13549737 & 0.03829791 \\
\hline$X_{\text {KIMBERA }}$ & 0.20521655 & 0.1537972 & 0.42237315 & 0.17885266 & 0.16143772 \\
\hline
\end{tabular}

Fuente: elaboración propia con datos del IPyC.

Análisis comparativo entre el modelo media-varianza y el modelo que incorpora la asimetría

El portafolio óptimo que se seleccionó al considerar el modelo media-varianza es el correspondiente al año 2012; mientras que para el modelo media-varianza-asimetría representado por la preferencia $(1,1,1)$ se seleccionó el portafolio correspondiente al año 2011. Para comparar ambos portafolios seleccionados es importante determinar la asimetría para cada portafolio con el objetivo de comparar los resultados obtenidos entre el modelo mediavarianza y el modelo multiobjetivo media-varianza-asimetría.

El cálculo de la asimetría de cada portafolio requiere de la suma de la multiplicación del coeficiente de asimetría de cada uno de los activos por el vector de pesos transpuestos. En los cuadros 7 y 8, se muestra la asimetría para el portafolio media-varianza que corresponde al año 2012, y el portafolio media-varianza-asimetría que corresponde al año 2011. 
Cuadro 7

Asimetría del portafolio media-varianza
Cuadro 8

Asimetría del portafolio media-varianza-asimetría

\begin{tabular}{|l|r|r|}
\hline \multicolumn{2}{|c|}{ Coeficiente de asimetría } & $\begin{array}{c}\text { Composición } \\
\text { de pesos }\end{array}$ \\
\hline \hline AMX L & -0.7661354 & 0.12787052 \\
\hline FEMSA UBD & 0.1444974 & 0.1071043 \\
\hline GFNORTE O & 0.1912817 & 0.0977222 \\
\hline WALMEX V & -1.9614212 & 0.11547424 \\
\hline TLEVISA CPO & -0.0412576 & 0.15495324 \\
\hline GMEXICO B & 0.0998969 & 0.03270177 \\
\hline CEMEX CPO & 0.2134851 & 0 \\
\hline ALFA A & 0.4487075 & 0.08746483 \\
\hline KOF L & -0.4857699 & 0.20309693 \\
\hline KIMBER A & 0.596847 & 0.07361197 \\
\hline \hline
\end{tabular}

Asimetría del portafolio

$-0.3088943$

\begin{tabular}{|l|r|r|}
\hline \multicolumn{2}{|c|}{ Coeficiente de asimetría } & $\begin{array}{c}\text { Composición } \\
\text { de pesos }\end{array}$ \\
\hline \hline AMX L & -0.190344 & 0 \\
\hline FEMSA UBD & 0.2068005 & 0.463629 \\
\hline GFNORTE O & -0.024012 & 0 \\
\hline WALMEX V & -0.104741 & 0 \\
\hline TLEVISA CPO & 0.2304384 & 0.062914 \\
\hline GMEXICO B & -0.275863 & 0 \\
\hline CEMEX CPO & 0.3353512 & 0 \\
\hline ALFA A & -0.434619 & 0 \\
\hline KOF L & -0.241317 & 0.051084 \\
\hline KIMBER A & 0.4505605 & 0.422373 \\
\hline \hline $\begin{array}{l}\text { Asimetría del } \\
\text { portafolio }\end{array}$ & 0.2883537 &
\end{tabular}

Fuente: elaboración propia con datos del IPyC.

El portafolio que considera el criterio media-varianza tiene una asimetría con sesgo negativo, bajo este escenario existe una mayor probabilidad de que se presenten rendimientos negativos en el futuro. Mientras que el portafolio que sigue como criterio de selección, la incorporación de la asimetría, muestra asimetría positiva, en este sentido la asimetría positiva indica que el portafolio tiene mayor probabilidad de obtener valores mayores a su promedio; por lo tanto, existe una mayor probabilidad de obtener rendimientos altos y así maximizar la función de utilidad del inversionista.

\section{Portafolio media-varianza-asimetría con diferentes combinaciones de preferencia del inversionista}

El cuadro 9 define los valores óptimos de la función multiobjetivo para diferentes combinaciones de preferencia para la media, la varianza y la asimetría. En el caso de las preferencias $(1,0,0)$, el 1 en la primera posición indica que se le da absoluta importancia al rendimiento, es decir, el inversionista desea 
maximizar su rendimiento. En el caso de las preferencias $(0,1,0)$, el máximo interés del inversionista es que el nivel de riesgo sea minimizado y para las preferencias $(0,0,1)$, el objetivo es obtener el valor óptimo de asimetría.

En el caso donde $\lambda_{\text {Rendimiento }} \lambda_{\text {Varianza }} \lambda_{\text {Asimetría }} \neq 0$ se consideraron las siguientes combinaciones de preferencias para el rendimiento, varianza y asimetría. $(1,1,1),(2,2,1),(1,2,1),(1,1,2),(1 / 2,1 / 4,1 / 4),(1 / 4,1 / 2,1 / 4),(1 / 4,1 / 4,1 / 2)$ y $(1 / 3,1 / 3,1 / 3)$, se obtuvieron los valores de la función multiobjetivo. Del conjunto de preferencias planteadas, la que minimizó la función de utilidad para cada portafolio anual, es la preferencia $(1 / 4,1 / 2,1 / 4)$ con un valor mínimo de 0.37032583 para el 2009, 2010=0.40419737, 2011=0.35488415, $2012=0.46597214$ y para el 2013 presentó un valor de 0.44462039 , los resultados pueden observarse en el cuadro 9.

\section{Cuadro 9}

Valores obtenidos al minimizar la función de utilidad con diferentes combinaciones de preferencias

\begin{tabular}{|l|l|l|l|r|r||}
\hline \multirow{2}{*}{ Preferencias } & \multicolumn{5}{|c|}{ Valor de la función de utilidad } \\
& $\mathbf{2 0 0 9}$ & $\mathbf{2 0 1 0}$ & \multicolumn{1}{c|}{$\mathbf{2 0 1 1}$} & \multicolumn{1}{c|}{$\mathbf{2 0 1 2}$} & $\mathbf{2 0 1 3}$ \\
\hline \hline$(1,0,0)$ & $1.6486 \mathrm{E}-16$ & 2.11824088 & $2.3969 \mathrm{E}-16$ & $1.5298 \mathrm{E}-16$ & $3.114 \mathrm{E}-16$ \\
\hline$(0,1,0)$ & $9.2594 \mathrm{E}-16$ & $2.0649 \mathrm{E}-08$ & $1.2493 \mathrm{E}-07$ & $1.0324 \mathrm{E}-08$ & $1.0639 \mathrm{E}-07$ \\
\hline$(0,0,1)$ & $3.8546 \mathrm{E}-16$ & $2.4159 \mathrm{E}-16$ & $2.4641 \mathrm{E}-16$ & 0 & $3.3315 \mathrm{E}-16$ \\
\hline$(1,1,0)$ & 0.67166596 & 0.54603088 & 0.61956201 & 0.56690705 & 0.87459454 \\
\hline$(1,1,1)$ & 1.40450674 & 1.4617535 & 1.168719 & 1.55847642 & 1.55364142 \\
\hline$(2,1,0)$ & 1.28140952 & 1.03097164 & 0.8998903 & 1.02959175 & 1.46241416 \\
\hline$(2,2,1)$ & 2.1258305 & 2.12419189 & 1.89861096 & 2.31545701 & 2.51321773 \\
\hline$(1,2,1)$ & 1.48129954 & 1.61678944 & 1.41953592 & 1.86388767 & 1.77848157 \\
\hline$(1,1,2)$ & 1.93029088 & 2.11824088 & 1.43822809 & 2.20674023 & 2.06227442 \\
\hline$(1 / 2,0,1 / 2)$ & 0.08187499 & 0.32481166 & 0.27050755 & 0.18319366 & 0.2882049 \\
\hline$(0,1 / 2,1 / 2)$ & 0.38058732 & 0.47421606 & 0.28979477 & 0.56493941 & 0.37932873 \\
\hline$(1 / 2,1 / 4,1 / 4)$ & 0.49732651 & 0.47926936 & 0.37197263 & 0.48424357 & 0.52320398 \\
\hline$(1 / 4,1 / 2,1 / 4)$ & 0.37032583 & 0.40419737 & 0.35488415 & 0.46597214 & 0.44462039 \\
\hline$(1 / 4,1 / 4,1 / 2)$ & 0.48257272 & 0.52956022 & 0.35955702 & 0.55168506 & 0.5155686 \\
\hline$(1 / 3,1 / 3,1 / 3)$ & 0.46816878 & 0.48725142 & 0.389573 & 0.51949214 & 0.51788054 \\
\hline \hline
\end{tabular}

Fuente: elaboración propia con datos del IPyC. 
Al considerar como marco de referencia que la preferencia que minimiza la función de utilidad para cada uno de los años es $(1 / 4,1 / 2,1 / 4)$, se obtuvo la composición de los pesos del portafolio. En el cuadro 10 por ejemplo se observa que los activos FEMSA UBD, TLEVISA CPO, KOF L, KIMBER A muestran un dominio sobre las proporciones de los pesos seleccionados, son activos que han sido seleccionados dentro de cada portafolio para cada año, por el contrario, el activo CEMEX CPO no muestra una participación significativa.

\section{Cuadro 10}

Composición de pesos del portafolio para el caso de preferencias $(1 / 4,1 / 2,1 / 4)$

\begin{tabular}{|c|c|c|c|c|c|}
\hline & 2009 & 2010 & 2011 & 2012 & 2013 \\
\hline$X_{\text {AMX L }}$ & 0.00538201 & 0.16528264 & 0.04352451 & 0.07787741 & 0 \\
\hline $\mathrm{X}_{\mathrm{FEMSA} \mathrm{UBD}}$ & 0.15503949 & 0.00041771 & 0.30863299 & 0.16916534 & 0.14098508 \\
\hline $\mathrm{X}_{\text {GFNORTE O }}$ & 0 & 0.0674176 & 0 & 0.09173669 & 0.08888711 \\
\hline $\mathrm{X}_{\text {WALMEX V }}$ & 0.20390841 & 0.16418183 & 0.00638667 & 0 & 0.08525573 \\
\hline $\mathrm{X}_{\text {TLEVISA CPO }}$ & 0.1784555 & 0.1333695 & 0.12493035 & 0.19405871 & 0.29125977 \\
\hline $\mathrm{X}_{\text {GMEXICO B }}$ & 0.00202411 & 0 & 0 & 0.07175001 & 0 \\
\hline $\mathrm{X}_{\text {CEMEX CPO }}$ & 0 & 0 & 0 & 0 & 0.124902 \\
\hline $\mathrm{X}_{\mathrm{ALFA} \mathrm{A}}$ & 0.05392057 & 0.19376169 & 0 & 0.09635768 & 0.02256755 \\
\hline $\mathrm{X}_{\mathrm{KOF} \mathrm{L}}$ & 0.14056951 & 0.13335676 & 0.14280906 & 0.16249792 & 0.13011082 \\
\hline $\mathrm{X}_{\text {KIMBER A }}$ & 0.26070039 & 0.14221226 & 0.37371642 & 0.13655624 & 0.11603194 \\
\hline
\end{tabular}

Fuente: elaboración propia con datos del IPyC.

En resumen, los resultados mostraron la efectividad de la optimización al utilizar un modelo de programación cuya función multiobjetivo representa la función de utilidad del inversionista mediante un polinomio que incorpora las preferencias del inversionista sobre la media, la varianza y la asimetría, el problema de portafolio con múltiples objetivos se solucionó de forma simultánea.

Se analizó el rol de preferencia por la asimetría como una variable objetivo en la selección de un portafolio de inversión óptimo. El argumento de aceptar que la distribución de los activos que conforman el portafolio de inversión no sigue una distribución normal, evidencia 
la importancia de analizar la incorporación de la asimetría en la determinación de un portafolio de inversión; en particular si la presencia de asimetría se presenta en todas las distribuciones de los activos analizados.

\section{Conclusiones}

Los resultados que se obtuvieron concuerdan con los que se reportan en los trabajos de Simkowit y Beedles (1978), Kane (1982), Lai (1991), Konno y Suzuki (1995), Chunhachinda, Dandapani , Hamid, y Prakash (1997) y Leung, Daouk, y Chen (2001). Las preferencias del inversionista entre la media, la varianza y la asimetría pueden ser fácilmente incorporadas al utilizar una programación de optimización multiobjetivo que minimiza la función de utilidad del inversionista. La ventaja de este enfoque es que es capaz de maximizar la aproximación mediante la asimetría para la expectativa de utilidad para cualquier aversión al riesgo en la función de utilidad.

Para los inversionistas con una absoluta aversión al riesgo, la asimetría de los rendimientos de un portafolio de inversión juega un papel importante dentro de la selección de un portafolio de inversión. Si se considera un inversionista que desea incrementar la asimetría es necesario hacer un significativo análisis de intercambio de la varianza, y rendimiento por asimetría, para obtener el mejoramiento de la asimetría. La importancia de este experimento es mostrar que el modelo es muy flexible y se puede utilizar de diferentes maneras dependiendo de las necesidades del inversionista.

La asimetría positiva es deseable dado que al incrementar la asimetría aumenta la probabilidad de rendimientos positivos y decrece la probabilidad de rendimientos negativos. El sesgo negativo en la asimetría incrementa la probabilidad de incurrir en pérdidas. Los resultados empíricos sugieren que la incorporación de la asimetría dentro de las decisiones de portafolio provoca un cambio importante en la construcción de un portafolio óptimo. 


\section{Referencias}

Arditti, F. (1971). Another look at mutual fund performance. Journal of Financial and Quantitative Analysis, 6(03): 909-912.

Chunhachinda, P.; K. Dandapani, S. Hamid y A. Prakash. (1997). “Portfolio selection and skewness": Evidence from international stock markets. Journal of Banking E Finance, 21 (2): 143-167.

Fama, E. (1965). "The behavior of stock-market prices". The journal of Business, 38(1):34-105.

Hanoch, G. y H. Levy (1970). "Efficient portfolio selection with quadratic and cubic utility". The Journal of Business, 43 (2): 181-89.

Kane, A. (1982). "Skewness preference and portfolio choice". Journal of Financial and Quantitative Analysis, 17 (1): 15-25.

Konno, H. y K. Suzuki (1995). “A mean-variance-skewness portfolio optimization model". Journal of the Operations Research Society of Japan, 38 (2): 173-187.

Lai, T. (1991). "Portfolio selection with skewness: a multiple-objective approach". Review of Quantitative Finance and Accounting, 1 (3): 293-305.

Leung, M.; H. Daouk y A. Chen (2001). “Using investment portfolio return to combine forecasts: a multiobjective approach". European Journal of Operational Research, 134 (1): 84-102.

Levy, H. y M. Sarnat (1972). Investment and portfolio analysis. New York: Wiley.

Markowitz, H. (1952). "Portfolio selection". The journal of finance, 7 (1): 77-91.

Prakash, A.; Chang, C. y T. Pactwa (2003). "Selecting a portfolio with skewness: Recent evidence from US, European, and Latin American equity markets". Journal of Banking \& Finance, 27 (7): 1375-1390.

Rubinstein, M. E. (1973). "The fundamental theorem of parameter-preference security valuation". Journal of Financial and Quantitative Analysis, 8 (01): 61-69.

Samuelson, P. (1970). "The fundamental approximation theorem of portfolio analysis in terms of means, variances and higher moments". The Review of Economic Studies, 37 (4): 537-542.

Simkowitz, M y W. Beedles (1978). "Diversification in a three-moment world". Journal of Financial and Quantitative Analysis, 13(5): 927-941.

Usta, I. y Y. Kantar (2011). “Mean-Variance-Skewness-Entropy Measures: A MultiObjective Approach for Portfolio Selection". Entropy, 13 (1): 117-133.

Zuluaga, L. y S. Cox (2010). “Improving skewness of mean-variance portfolios". North American Actuarial Journal, 14 (1): 59-67. 